\title{
NUEVAS TENDENCIAS EN LA HISTORIOGRAFÍA ESPAÑOLA
}

\author{
ISRAEL SANMARTÍN \\ Universidad de Santiago de Compostela
}

\begin{abstract}
Resumen
El artículo se enmarca en lo que algunos historiadores han denominado "historiografía inmediata", que consiste en reflexionar sobre la historia de la historiografía que se está desarrollando a la par del propio historiador. La investigación describe, analiza y explica la evolución de la historiografía española reciente en los últimos años. Así, el autor tratará en su primer apartado las características de la historiografía española reciente, después identificará las últimas tendencias historiográficas españolas, para terminar con la profundización en una de ellas; Historia a Debate, que servirá de antesala para las conclusiones.
\end{abstract}

\section{Palabras clave}

Historiografía española, historiografía, Historia a Debate, Memoria histórica, tendencias, Tomas S. Kuhn, comunidad científica, paradigma, red académica.

\begin{abstract}
The article is framed in which some historians have denominated "immediate historiography" (it consists of reflecting on the history of the historiography developed on the time of the historian. The investigation in the describes, analyzes and explains the evolution of the recent Spanish historiography. Thus, the author will studie ithe characteristics of the recent Spanish historiography, the last historiographi Spanish tendencies, and will deepen one of them; History to Debate, that will serve as waiting room for the conclusions.
\end{abstract}

\section{Keywords}

Spanish historiography, historiography, History under Debate, historical Memory, tendencies, Tomas S. Kuhn, scientific community, paradigm, academic network. 
Argumentar sobre la historiografía española y gallega en los últimos años es una labor que requiere tanto de un conocimiento sobre la historia que se está escribiendo e investigando en toda la Península Ibérica, como de una actualización teórica y metodológica que permita reconocer y definir las diferentes tendencias que han ido surgiendo. Con el objetivo de cumplir con estas dos premisas, este trabajo partirá de lo que ha sido denominado por algunos historiadores españoles como historiografía inmediata $^{1}$, que consiste en reflexionar sobre la historia de la historiografía que se está desarrollando a la par del propio historiador, que es determinada por la historia de la que ambos, la historiografía y el historiador, son testigos, y que se caracteriza por una mayor implicación del historiador en los hechos historiográficos ${ }^{2}$, por la provisionalidad de sus conclusiones -que han de permanecer abiertas-, y por la necesidad de considerar el contexto ${ }^{3}$. Además de la historiografía inmediata, otro de los ejes de este pequeño trabajo será el de desarrollar la destreza de "reconocer tendencias"4, con el objeto de ofrecer una panorámica de las diferentes tendencias y líneas de investigación de la historiografía española en los últimos diez años. Todo con la base de desarrollar una panorámica subjetiva ${ }^{5}$ y que se encuadrará en lo que Merton denominó Middle Theories, es decir, un trabajo no sólo empírico pero tampoco únicamente teórico, que son los polos en los que suelen circular los trabajos de esta naturaleza. El objetivo no es otro que adoptar una postura teórica en consonancia con la sociedad en la que se

\footnotetext{
${ }^{1}$ Véase Carlos Barros en Carlos Barros. Ver "El retorno de la historia", Actas del II Congreso Internacional Historia a debate. I. Cambio de siglo, Santiago, 2000, pp. 153-173; y Gonzalo Pasamar en sus diferentes intervenciones en el III Congreso Internacional Historia a Debate.

${ }^{2}$ El historiador es sujeto de la historia a la vez que intérprete e investigador. Ver punto I "ciencia con sujeto" del Manifiesto historiográfico Historia a Debate, Santiago de Compostela, 2004, p. 3.

3 "La historiografía inmediata que preconizamos -y que practicamos desde hace años- observa e interpreta de dónde viene y adónde va la historia que se escribe, se compromete con críticas y propuestas de futuro, y aún debería reflexionar sobre el sentido de la historia en colaboración/competencia con los filósofos inmersos en el tiempo presente, del mismo modo que tenemos que competir/colaborar con los periodistas en el análisis de la historia inmediata y con los escritores en el enfoque y la difusión de los relatos históricos. ¿Por qué el historiador no ha de meditar, yendo de lo concreto a lo abstracto y viceversa, las implicaciones teóricas de la historia y de la historiografía? ¿Por qué la teoría vinculada a la historia inmediata hay que dejársela a los filósofos políticos? ¿No sería mejor una convergencia de historiadores, filósofos, politólogos, sociólogos y periodistas en la interpretación, cada uno desde su ángulo de análisis, de los grandes hechos de la historia inmediata (y, si se quiere, no inmediata)?", escribe Carlos Barros. Ver "El retorno de la historia", Actas del II Congreso Internacional Historia a debate. I. Cambio de siglo, Santiago, 2000, pp. 153-173.

${ }^{4}$ Punto IX del Manifiesto historiográfico Historia a Debate, Santiago de Compostela, 2004.

${ }^{5}$ En el sentido de que es imposible conocer todos los trabajos que se están desarrollando o que se han publicado en España en los últimos diez-quince años. Ver punto I "Ciencia con sujeto" del Manifiesto historiográfico Historia a Debate, Santiago de Compostela, 2004, p.3.
} 
desarrolla y evitar la explicación de la historiografía española sólo a partir de un segmento de la totalidad ${ }^{6}$.

Siguiendo estos ejes metodológicos, el artículo tratará en su primer apartado las características de la historiografía española de los últimos años, después se pasará a identificar las últimas tendencias historiográficas españolas, para a continuación centrarnos en una de ellas, Historia a Debate. A modo de conclusión identificaremos algunos puntos comunes y estableceremos algunas consideraciones para el futuro de la historiografía española.

Antes de comenzar con labor citada, empezaremos por exponer el estado de la historiografía española, que se puede pulsar empíricamente a través de la Encuesta Internacional el Estado de la Historia ${ }^{7}$, que se envió a más de 30.000 historiadores (además de estar más de un año colgada de Internet) y que contestaron un total de 605 (de ellos 255 eran españoles). Una de las conclusiones de la Encuesta es el buen momento de la historiografía española y su creciente consideración e influen$\mathrm{cia}^{8}$ (Ver tabla 1), que es una de las premisas con las que se desarrollará este trabajo. Pese a este diagnostico previo, también se considerarán otras realidades actuales de la historiografía, como la percepción de que el marco del Estado-nación ya no es el más adecuado para el desarrollo de la historia y de la historiografía ${ }^{9}$; así como la excesiva valoración positiva de la fragmentación actual de la disciplina ${ }^{10}$, y la constatación, todavía, del intercambio desigual entre las historiografías ${ }^{11}$.

\footnotetext{
${ }^{6}$ Ver PEREIRA-MENAUT, G., "Presentación" en PEREIRA-MENAUT, G., La economía de los romanos, La editorial de la historia, Santiago de Compostela, 1994, p. XIII.

${ }^{7}$ Que fue realizada por un equipo de investigadores de diferentes áreas y universidades coordinado por el profesor Carlos Barros, y al amparo de dos proyectos de investigación financiados por la Xunta de Galicia entre los años 1996-2001. Para ver los datos, cuestionario y resultado: http://www.hdebate.com/encuesta/menu.htm

${ }^{8}$ Ver resultados en http://www.h-debate.com/encuesta/resultados/e_comunidad/p38.htm

${ }^{9}$ Ver pregunta 44 de la Encuesta Internacional "El Estado de la historia". En ella se concluye que el $49 \%$ de los encuestados contestaban que el marco más adecuado para el desarrollo de la historia y de la historiografía ya no era el estado nación; por 25\% que sí; el resto no contestaban. Hay que considerar que la gran cantidad de "NS/NC" (no sabe, no contesta) aparecen porque la encuesta se podía cubrir por Internet en tres partes, y mucha gente no las cubrió todas. Ver gráfico y resultados en http://www.h-debate.com/encuesta/resultados/e_fragmentacion/p44/p44.htm).

${ }^{10}$ En cuanto a la pregunta ¿Está de acuerdo con la actual fragmentación de temas, métodos y especialidades históricas?, un total de un $32 \%$ estba de acuerdo frente a un $46 \%$ que no Ver resultados en http://www.h-debate.com/encuesta/resultados/e_fragmentacion/p42/p42.htm

${ }^{11}$ Ver resultados en http://www.h-debate.com/encuesta/resultados/e_comunidad/p37/p37_1_1.htm
} 


\section{ALGUNAS PARTICULARIDADES DE LA HISTORIOGRAFÍA ESPAÑOLA}

Después de esbozar la situación de partida de la historiografía española, veamos ahora qué hay detrás de esos datos, y para ello vamos a hacer un poco de historia. No es ningún secreto que la historiografía española ha estado supeditada al desarrollo de los acontecimientos políticos del país, que provocaron cambios historiográficos de amplio calado al menos en dos ocasiones. Una primera alteración, involucionista, se produjo tras la Guerra Civil española, cuyo desencadenamiento provocó un notable estancamiento metodológico e historiográfico ${ }^{12}$, mientras la historiografía europea continuaba con su evolución natural hacia la llamada "revolución historiográfica del siglo $\mathrm{XX} " 13$. La segunda ruptura, de adecuación, se produjo como consecuencia de la transición democrática, momento en el cual la historiografía española intenta homologarse a las nuevas tendencias de las grandes historiografías europeas (marxismo y Annales) ${ }^{14}$. En ese proceso la historiografía española experimentó una eclosión plural en contenidos y métodos que la llevaron a superar el aislamiento y retraso que lastraba ${ }^{15}$.

Estas rupturas y discontinuidades ${ }^{16}$ provocaron en la historiografía española una tardía recepción de la revolución historiográfica del siglo $\mathrm{XX}^{17}$, una ausencia de

${ }^{12}$ El profesor Gonzalo Pasamar, uno de los especialistas más importantes sobre historiografía española, denomina a este período como "ruptura con la tradición liberal". Ver PASAMAR, G., Historiografía e ideología en la posguerra española: la ruptura de la tradición liberal, Universidad de Zaragoza, Zaragoza, 1991. Gonzalo Pasamar, Ignacio Peiró y otros miembros de su grupo, como Miguel Marín, han investigado e investigan con una solvencia sobresaliente la historiografía española.

${ }^{13}$ Especialmente durante el período de entreguerras. La "revolución historiográfica del siglo XX" está ausente en la historiografía española. Ver OLÁBARRI, I., "La recepción en España de la revolución historiográfica del siglo XX", en AA. VV., La historiografía en Occidente desde 1945, Eunsa, Pamplona, 1985, pp. 87-109.

14 “Una vez restaurada la democracia, y la monarquía, la renovación historiográfica no enlaza con la tradición liberal-positivista sino que parte de las nuevas bases: las creadas por las nuevas tendencias internacionales, Annales y marxismo, que atraviesan los Pirineos.".Ver BARROS, C., "Inacabada transición de la historiografía española", Bulletin d'Histoire Contemporaine de l'Espagne, $\mathrm{n}^{\circ} 24$. Bourdeaux, décembre 1996, pp. 469-493. Ver también BARROS, C., "El retorno del sujeto social en la historiografía española", Estado, protesta y movimientos sociales, Zarautz, 1998, pp. 191-214.

${ }^{15}$ Garzón llama a esto la "edad de plata" de la historiografía española. Ver PÉREZ GARZÓN, Juan Sisinio, "Sobre el esplendor y la pluralidad de la historiografía española", en AA.VV, Tuñón de Lara y la historiografía española, Alianza, Madrid, 1999, pp. 335-337. Ver también TUÑÓN DE LARA, M., Historiografía española contemporánea, Siglo XXI, Madrid, 1980.

${ }^{16}$ La evolución de la historiografía española en "rupturas" y "continuidades" reafirma lo adecuado de la explicación de Carlos Barros al respecto.

${ }^{17}$ Carlos Barros señala que lo largo de 1975 un grupo de historiadores jóvenes, y menos jóvenes, escriben sobre la situación y perspectivas de la historia, en el Boletín Informativo de la Fundación Juan March, en base a las tendencias en aquel momento: Annales (Antonio Eiras Roel, José Ángel García de 
movimientos pendulares extremos, y una historiografía con poca proyección fuera de la Península. Con este contexto, se pueden distinguir una serie de características en la historiografía española desde los años ochenta: ${ }^{18}$

a) Hegemonía de la historia social (con gran diversidad temática y deuda metodológica anglosajona) ${ }^{19}$.

b) Eclosión de los estudios locales, promovidos tanto desde la universidad como desde los centros locales de investigación, ayuntamientos y otras instituciones públicas y privadas, que han provocado, paralelamente, del florecimiento de eruditos locales fruto de la desprofesionalización de la historia. Hay que reseñar aquí la falta de historia comparada entre las diferentes regiones y localidades y la ausencia de estrategias globales y conjuntas de investigación ${ }^{20}$.

c) Ausencia de escuelas metodológicas y de debates teóricos ${ }^{21}$, aunque sería injusto no reconocer la labor realizada al respecto por los diferentes libros y congresos publicados al respecto (en los que hay que incluir a Historia a Debate). Pese a todo, no es suficiente.

d) Fragmentación temática y microespecialización. Este rasgo distintivo es uno de los más preocupantes puesto que la fragmentación no tiene reverso y conlleva una difícil vuelta atrás. Cada vez más los historiadores se centran en su especialidad temática olvidando la reflexión general, empírica y teórica.

e) Continuación de una gran tradición de historia positivista. Carlos Barros ha señalado que muchos historiadores han entendido como una salida a la crisis de la historia la vuelta a los archivos y la realización de investigaciones en exceso empíricas, con lo que dan la espalda definitivamente a la disciplina.

Cortázar), marxismo (Juan José Carreras, Antonio Elorza) e historiadores tradicionales (Luis Suárez, José María Jover). Ver BARROS, Carlos, "El paradigma común de los historiadores del siglo XX", Estudios Sociales. Revista universitaria semestral, no 10, Santa Fe (Argentina), 1996, pp. 21-44.

${ }^{18}$ Para algunas de ellas, ver PÉREZ GARZÓN, Juan Sisinio, "Sobre el esplendor y la pluralidad de la historiografía española", en AA.VV, Tuñón de Lara y la historiografía española, Alianza, Madrid, 1999, pp. 343-347.

${ }^{19}$ Ver VÁZQUEZ, Francisco, "La historia social española y los nuevos paradigmas: encuentros y desencuentros", en Actas del II Congreso Internacional Historia a Debate, Vol. I, Santiago de Compostela, 2000, pp. 219-230.

${ }^{20}$ Aquí ha habido algunas novedades como las historias locales transdisciplinares y multiárea, como se puede observar en los trabajos del investigador del CSIC, Isidro García Tato. Como ejemplo ver las más de 1400 páginas del voluminoso Valdeorras de cara al año 2000. Pasado, presente y futuro de una comarca, 2 vols., Caixa Ourense-Instituto de Estudios Valdeorreses, O Barco de Valdeorras, 1996.

${ }^{21}$ Ver SANTANA, J. M., "Globalización e historiografía", en Actas II Congreso Internacional Historia a Debate, Santiago de Compostela, pp. 93-103. 
f) Importancia del postmodernismo, que en la práctica se ha quedado en una preocupación teórica, de debate (sobre todo de divulgación de lo que aparece en el ámbito anglosajón) ${ }^{22}$, y de las relaciones entre historia y narración ${ }^{23}$. $\mathrm{Su}$ verdadera coartada ha sido la de promover un ambiente donde el "todo vale" se ha hecho un sitio y en el que las propuestas individuales parecer prevalecer sobre los proyectos y estrategias colectivas.

g) Historia inmediata. El fomento de la historia reciente, historia inmediata, historia del tiempo presente, historia actual ${ }^{24}$ o historia vivida ${ }^{25}$, según diferentes denominaciones ${ }^{26}$, es otra de las características de la nueva historiografía española. Desde Historia a Debate se han impulsado diferentes investigaciones al respecto además de discutir diariamente sobre la cuestión en la red a través de lista de "Historia Inmediata"27. La Historia Inmediata de HaD supone historiográficamente la culminación de un lento y difícil proceso de incorporación del tiempo presente al ámbito de trabajo de los historiadores.

22 Algunos de los historiadores españoles que mejor han entendido la relación entre postmodernismo e historia han sido Miguel A. Cabrera, Jaume Aurell y José C. Bermejo (BERMEJO, J.C., "On History Considered as Epic Poetry", History and Theory, Vol. 44, n², may 2005, pp. 182-194; BERMEJO, J. C., "Making histhory, talking about history", History and Theory, n 20, may 2001, pp. 190205; BERMEJO, J.C., "Los historiadores: sus textos, sus métodos y el problema del pensamiento", Gallaecia, 24, pp. 265-279; BERMEJO, J.C., "Los historiadores y la búsqueda de la certeza", Agora, 23/1, pp. 161-181). Ver CABRERA, M. A., "La situación actual de la historia: un paisaje cambiante" en Miguel A. Cabrera y Marie McMahon (coords.), La situación de la Historia. Ensayos de historiografía, Universidad de La Laguna, Tenerife, 2002, pp.13-52 y sobre todo: CABRERA, M. A., Historia, lenguaje y teoría de la sociedad, Madrid, 2001; Aurell, Jaume, La escritura de la memoria. De los positivismos a los postmodernismos, Universidad de Valencia, Valencia, 2005. A estos dos autores habría que sumarle Francisco Vázquez, ver VÁZQUEZ, Francisco, "La historia social española y los nuevos paradigmas: encuentros y desencuentros", en Actas del II Congreso Internacional Historia a Debate, Vol. I, Santiago de Compostela, 2000, pp. 219-230.

${ }^{23}$ Ver VÁZQUEZ GARCÍA, F., "La controversia sobre historia y narración: un espectro epistemológico", Miguel A. Cabrera y Marie McMahon (coords.), La situación de la Historia. Ensayos de historiografía, Universidad de La Laguna, Tenerife, 2002, pp. 89-110; VÁZQUEZ GARCÍA, F., "La transformación contemporánea de la hermenéutica y el estatuto epistemológico de la historia", Fragmentos de Filosofía, 2, 1992, pp. 165-184; J. C. BERMEJO y P. Piedras, Genealogía de la historia. Ensayos de historia teórica III, Akal, Madrid, 1999; MORALES MOYA, Antonio, "Historia y postmodernidad", Ayer, nº, 1992, pp. 15-38.

${ }^{24}$ Grupo de investigación y Asociación dirigida por el profesor Julio Pérez Serrano de la Universidad de Cádiz (ver http://www.historia-actual.com)

${ }^{25}$ ARÓSTEGUI, Julio, La historia vivida. Sobre la historia del presente, Alianza, Madrid, 2004.

${ }^{26}$ Las diferentes denominaciones esconden detrás un entendimiento tanto historiográfico como metodológico diferente, que queda fuera del alcance de este trabajo. En esta investigación siempre nos referiremos a la historia inmediata como la entendemos desde Historia a Debate.

27 Ver especialmente el debate “¿Es posible una historia inmediata?”, que se inició el 15 de noviembre del año 2000, ver http://www.h-debate.comlSpanishla_hinmediata.htm 
h) Importancia del hispanismo y de los hispanistas. Por ejemplo, como indica Gonzalo Pasamar para el caso de los contemporaneistas, todavía en algunas revistas, como la Journal of Contemporary History, la historia contemporánea de España sólo puede observarse bajo la óptica de los hispanistas anglosajones. Para Pasamar la escasa presencia internacional de la historiografía española se debe a la incapacidad de la historiografía española para participar en los debates teóricos internacionales (auque esta situación está cambiando) y la falta de epistemólogos y de teorizadores ${ }^{28}$.

i) El avance de la historiografía española se constata en la capacidad de los historiadores de hacer estados de la cuestión sobre el de desarrollo y actualidad de la historiografía internacional ${ }^{29}$, además de hacerse visibles con la publicación de artículos en las grandes revistas internacionales ${ }^{30}$.

j) Hay otras características que también se deben reseñar, como el cierto aislamiento de las diferentes historiografías nacionales peninsulares; el predominio del contemporaneismo; la endogamia; la dependencia de las celebraciones (el uso público de la historia); la poca interdisciplinaiedad; atraso en la recepción de las nuevas historias y escaso arraigo de algunas de ellas; creci-

\footnotetext{
${ }^{28}$ Ver PASAMAR, G., La historia contemporánea. Aspectos teóricos e historiográficos, Síntesis, Madrid, 2000.

${ }^{29}$ Algunos ejemplos son: CASADO QUINTANILLA, Blas (coord.), Tendencias historiográficas actuales, UNED, Madrid, 2001; HERNÁNDEZ SANDOICA, Elena, Tendencias historiográficas actuales, Akal, Madrid, 2004; Miguel A. Cabrera y Marie McMahon (coords.), La situación de la Historia. Ensayos de historiografía, Universidad de La Laguna, Tenerife, 2002; CARRERAS, Juan J., Razón de historia. Estudios de historiografía, Prensas Universitarias de Zaragoza, Zaragoza, 2000; Carlos Forcadell e Ignacio Peiró (Coords.), Lecturas de la Historia. Nueva reflexiones sobre historia de la historiografía, Instituto Fernando el Católico, Zaragoza, 2001; Aurell, Jaume, La escritura de la memoria. De los positivismos a los postmodernismos, Universidad de Valencia, Valencia, 2005; AA.VV, Historia: ¿Principio del fin o fin del principio?, Madrid, 2002; Elena Hernández Sandoica y Alicia Langa, Sobre la historia actual entre política y cultura, Abada Editores, Madrid, 2005 (doy las gracias al profesor Santiago Jiménez de la Universidad de Santiago de Compostela por ponerme sobre la pista de esta publicación); y el muy interesante y audaz SANTANA, Juan M., Paradigmas Historiográficos Actuales, Fundación Buría, Barquisimeto (Venezuela), 2005. Por último, es de imprescindible presencia en esta lista, subjetiva, los libros: BERMEJO, J.C., Entre Historia y filosofía, Akal, Madrid, 1994; BERMEJO, J. C., ¿Qué es la historia teórica?, Akal, Madrid, 2004.

${ }^{30}$ Por ejemplo: BARROS, C., "The history ahead", Storia della Storiografia, no 30, 1996, pp. $77-$ 106; BERMEJO, J.C., "On History Considered as Epic Poetry", History and Theory, Vol. 44, n², may 2005, pp. 182-194; BERMEJO, J. C., "Making histhory, talking about history", History and Theory, $\mathrm{n}^{\circ}$ 20, may 2001, pp. 190-205. CABRERA, M. A., "Linguistic aproach or return to subjetivism? In search of an alternative to social history", Social History, Vol. 24, 1, 1999, pp. 74-89; PEREIRAMENAUT, Gerardo, "Che cos`è un munus?", Athenaeum. Studi di Letteratura e Storia dell 'Antichità, 2004, pp. 169-215; y el libro History under Debate. International Reflection on the Discipline, The Haworth Press, New York, 2004.
} 
miento notable de la producción y publicación; ausencia de grandes escuelas y necesidad de importar de historiografías más avanzadas; renovación de la historia política y de las relaciones internacionales (ausente durante el franquismo); auge y autonomía de la nueva historia económica; escasez de historia comparada; poca dedicación de los historiadores españoles a la historia de otros estados; penuria teórica y metodológica; falta de integración de la historiografía anglosajona y española ${ }^{31}$ a pesar, como se ha señalado, de que se están dando pasos en sentido contrario; intensificación de las relaciones personales exteriores en detrimento de las interiores ${ }^{32}$; falta de trabajo colectivo y limitada innovación técnica ${ }^{33}$.

k) Precariedad de los jóvenes investigadores e indefinición institucional de la disciplina dependiente del nuevo marco educativo europeo y su convergencia.

Además de estos rasgos identitarios, en cuanto a métodos y enfoques hay que considerar la cuestión de la especialización en áreas, donde cada una tiene sus propias peculiaridades ${ }^{34}$. Y hablando de áreas, parece que continúa la patrimonialización que los investigadores de historia contemporánea realizan sobre la historiografía, metodología y teoría de la historia ${ }^{35}$.

Por su perfil identificativo y por sus carencias, algunos historiadores como Carlos Barros, hablan de la "inacabada la transición historiográfica española” a media-

${ }^{31}$ GRANJA, José L. de la, "La historiografía española reciente: un balance", Actas del I Congreso Internacional Historia a Debate, Vol. I, pp. 299-307.

${ }^{32}$ Aunque si se constata empíricamente, el dato no está tan claro, ver las respuestas al particular en la Encuesta Internacional El estado de la hisgtoria, ver: http://www.h-debate.com/encuesta/resultados/ e_comunidad/p36/p36_6.htm y http://www.h-debate.com/encuesta/resultados/e_otrosdatos/pc/pc3.htm

33 IRADIEL, P., "El futuro de la historiografía española", en "Mesa R. El futuro de la historiografía española”, Actas II Congreso Internacional Historia a Debate, Santiago de Compostela, 2000, pp. 340-342.

${ }^{34}$ Ver los trabajos de José M. Blázquez (Antigua), Emilio Mitre (Edad Media), Fernando Sánchez Marcos (Edad Moderna) y José M. Cuenca (Contemporánea) en GALLEGO, José A. (coord), Historia de la historiografía española, Ediciones Encuentro, Madrid, 1999, p. 300-302. En cuanto a la la historiografía contemporánea, ver HERNÁNDEZ SANDOICA, E., "La historia contemporánea en España: tendencias recientes", Hispania, n 198, 1998, pp. 65-95. Para la situación de la historia antigua ver DUPLÁ, A., "El futuro de la historiografía española", en "Mesa R. El futuro de la historiografía española", Actas II Congreso Internacional Historia a Debate, Santiago de Compostela, 2000, pp. 337-339; Antonio Duplá y Amalia Emborujo, Estudios sobre historia antigua e historiografía moderna, Universidad de País Vasco, Vitoria, 1994; DUPLÁ, A., "Historia Antigua y franquismo. Ensayo historiográfico", en C. Forcadell, I. Peiró (eds.), Lecturas de la Historia. Nueve reflexiones sobre Historia de la Historiografía, Zaragoza, Inst. Fdo. el Católico, 2001, 167-190. Para un trabajo renovador en la historia antigua, consultar: PEREIRA-MENAUT, Gerardo (dir.), La economía política de los romanos, Vol I y Vol II, Arcana-Veri, Santiago de Compostela.

${ }^{35}$ Aunque cada vez hay más excepciones: Aurell, Barros, Bermejo, Duplá, Mitre, etc. 
dos de los años $90^{36}$. Esa transición está alimentada por una crisis ${ }^{37}$ de la disciplina a nivel social y académico.

\section{LAS NUEVAS TENDENCIAS DE LA HISTORIOGRAFÍA ESPAÑOLA}

Volviendo a la transición historiográfica, parece que se está empezando a cerrar con el dinamismo y la autonomía de la historiografía española en la última década, que se ha visto reflejado en la aparición de tres tendencias historiográficas actuales y globales, es decir, transversales en cuanto a áreas y grupos de investigación, que no representan ni los intereses de toda la comunidad de historiadores ni los de toda la historiografía española, sino a la percepción que tenemos desde Historia a Debate.

a) La idea histórica de España ${ }^{38}$, que podría ser llamada también "historias nacionales". Comenzó con García de Cortázar en su Breve historia de Espa$\tilde{n} a^{39}$ y ha seguido evolucionando, llegando a ser utilizada por el gobierno del Partido Popular como reclamo intelectual de lo que algunos han denominado, sobre todo desde posturas nacionalistas, "nacionalismo español" y que se basa en la excesiva proyección de la corona de castilla sobre el resto del territorio con el objetivo de expresar una historia de España en exceso uniforme, excluyente con gran parte de los pueblos de España y poco plural. Este proyecto intelectual nace como reacción a la potencialización que habían promovido las Comunidades Autónomas de sus historias nacionales respectivas ${ }^{40}$, que mues-

\footnotetext{
${ }^{36}$ Ver BARROS, C., "Inacabada transición de la historiografía española”, Bulletin d'Histoire Contemporaine de l'Espagne, $\mathrm{n}^{\circ}$ 24. Bourdeaux, décembre 1996, pp. 469-493. En ese diagnóstico coincide con Pasarmar, quien habla de que en la historiografía española falta por completar el "proceso de innovación", ver Ver PASAMAR, G., La historia contemporánea. Aspectos teóricos e historiográficos, Síntesis, Madrid, 2000, p. 248.

${ }^{37}$ Entiéndase "crisis" como el cambio de una situación a otra y no como un mero recurso recurrente, al estilo de la "crisis de la historia como ideología", etc.

${ }^{38}$ Uno de los primeros que propuso la necesidad de esta línea de trabajo fue Carlos Barros, aunque no se respetó su idea, que era mucho más plural y abierta. Ver BARROS, C., "Inacabada transición de la historiografía española", Bulletin d'Histoire Contemporaine de l'Espagne, $\mathrm{n}^{\circ} 24$. Bourdeaux, décembre 1996, pp. 469-493.

39 GARCÍA DE CORTÁZAR, F. y GONZÁLEZ VESGA, José M., Breve historia de España, Alianza, Madrid, 1994.

${ }^{40}$ Para el caso catalán ver el artículo RIQUER, Borja de, "Panorámica actual de la historiografía catalana"; para el caso vasco GRANJA, José Luis de la, "La nueva historiografía vasca" y para la historiografía gallega BERAMENDI, Justo G., "La historiografía gallega",y para otros SUÁREZ, Manuel, "La pequeña España. Particularismo centrípeto e historiografía contemporánea desde la transición democrática", todos en AA.VV, Tuñón de Lara y la historiografía española, Alianza, Madrid, 1999, pp. 279-304.
} 
tran los mismos problemas de excesiva uniformidad y manipulación e invención históricas. Este proceso ha ido en los últimos años radicalizándose aunque venía desarrollándose desde tiempo atrás ${ }^{41}$. La apertura de un nuevo proceso político constituyente con la reforma de los Estatutos de las Comunidades Autónomas y, quizá, con la apertura del debate sobre la reforma de la Constitución son simplemente un episodio más de este proceso. El objetivo es lograr un ajuste, que no un enjuague; y un equilibrio que no una negociación, de todas las realidades nacionales peninsulares donde la matriz no sea un excesivo peso castellano ni de otras naciones, con el fin de superar, lo que muy acertadamente José Andrés Gallego ha llamado "el problema de España"42.

b) La recuperación de la memoria histórica, que es un intento de recuperación de la memoria histórica de la Guerra Civil mediante la contribución de "donantes de memoria" y de los restos humanos de las víctimas ${ }^{43}$ con el objetivo de reconstruir la historia de los derrotados. Se trata de investigar la historia de los vencidos desde los testimonios, su realidad inmediata y su contexto general, para reconstruir una realidad que no ha podido ser escrita todavía ${ }^{44}$.

La cuestión de la recuperación de la memoria histórica es parte de otra línea de investigación que ha renacido últimamente en España, que es la Guerra Civil. De tal forma, siguiendo los bandos de la Guerra, los historiadores se dividen entre los de un bando y los de otro, donde lo que unos ven blanco otros lo visualizan negro. En ese mismo dogmatismo unos afirman que la guerra empezó en el 34, otros en el 32; y ambos lados de la trinchera disparan un número de víctimas y unas explicaciones de las causas, desarrollo y consecuencias totalmente opuestas. Se enfrentan lo que

${ }^{41}$ La revista Manuscrits publicó a mediados de los 90 un especial sobre los mitos de las diferentes historiografías nacionales. Manuscrits. Revista d'història moderna, no 12, Barcelona, 1994. Ver también el sugerente artículo: PEREIRA-MENAUT, G., "Sobre la función del pasado histórico en los movimientos nacionalistas" en Homenaje a F. Presedo, Sevilla, 1994, pp. 852-862.

42 GALLEGO, José A., "El problema (y la posibilidad) de entender la historia de España" en GALlEGO, José A. (coord), Historia de la historiografía española, Ediciones Encuentro, Madrid, 1999, p. 300-302

43 Desde mucho antes ya se habían iniciado investigaciones sobre la represión franquista. Ver PRESTON, P., "La historiografía de la Guerra Civil española: de Franco a la democracia", en AA.VV, Tuñón de Lara y la historiografía española, Alianza, Madrid, 1999, pp. 161-166.

${ }^{44}$ Destaca en esta labor la Asociación para la recuperación de la memoria histórica. En esta tendencia es muy importante la diferenciación entre los conceptos de memoria e historia y la explicitación de ambos conceptos siempre es precisa ante cualquier investigación. Para ver un artículo donde se relacionan ambas, ver SPIEGEL, Gabrielle M., "Memoria e historia: tiempo litúrgico y tiempo histórico" en Miguel A. Cabrera y Marie McMahon (coords.), La situación de la Historia. Ensayos de historiografía, Universidad de La Laguna, Tenerife, 2002, pp.53-69. 
se conoce como tesis franquistas contra las tesis académicas; unas avaladas desde los propagandistas y los historiadores ajenos a la academia y las otras desde dentro de las universidades. Han sido muy significativos los debates sostenidos por Pío Moa, César Vidal, José María Marco o Ricardo de la Cierva contra Moradiellos, Preston, Juliá, Tusell, Serna, Reig, etc. en las páginas de los principales diarios españoles tanto digitales (libertaddigital.com, rebelion.org, periodistadigital.com) ${ }^{45}$ como impresos (El Mundo, El País, La Vanguardia, ABC y La Razón), en las revistas especializadas (Revista de Libros, El Catoblepas, etc.), así como en la comunidad internacional de historiadores Historia a Debate (www.h-debate.com).

c) Historia a Debate, que es comunidad-red de historiadores que ofrecen desde una postura pluridisciplinar, multiárea y multinacional, con base española y latina, una alternativa historiográfica global desde una base latina.

La alternativa historiográfica de Historia a Debate ${ }^{46}$, se caracteriza por: a) combinación, y atención permanente, de la reflexión con la investigación empírica, con la intención de buscar una historia mestiza, mixta y global y evitar la fragmentación; b) democratización, más allá de situación profesional y especialidad temática, en la posibilidad de investigar, reflexionar y debatir sobre historiografía, metodología y teoría de la historia ; c) debate permanente; d) historiografía inmediata. Interés por la metodología, la historiografía y la teoría de la historia del pasado, presente y futuro ${ }^{47}$.

Estas tres tendencias tienen unas características comunes: a) desbordan la dimensión de un mero equipo o grupo de investigación, constituyendo corrientes historiográficas en las cuales participan historiadores de áreas y especialidades diferentes; b) responden a acciones académicas españolas -o latinas de origen español- con perfiles propios, no miméticas, aunque conectadas con las grandes tendencias europeas y estadounidenses; c) configuran tres formas diferentes de recuperación y actualización de la aspiración al compromiso ético, social y político de los historiadores con su tiempo ${ }^{48}$.

\footnotetext{
${ }^{45}$ Ver la reciente polémica entre Pío Moa y Justo Senra en el diario digital Periodistadigital.com. Ver http://blogs.periodistadigital.com/24por7.php/2005/11/12/justo_serna_vs_pio_moa_duelo_de_titanes

${ }^{46}$ Que engloba la historia mixta y la historia inmediata. BARROS, C., "La historia mixta como una historia global", <http://www.h-debate.com/cbarros/spanish/articulos/historia_medieval/mixta.htm>, [consulta 7 de noviembre de 2005].

47 BARROS, C., "Defensa e ilustración del Manifiesto historiográfico de Historia a Debate", Revista d'Història Medieval, Valencia, $\mathrm{n}^{\circ}$ 12, 2001-2002, pp. 389-433.

48 BARROS, C., "Primeras conclusiones del III Congreso Internacional Historia a Debate (14-18 de julio de 2005)", E-l@atina. Revista electrónica de estudios latinoamericanos, UDISHAL, Universidad de Buenos Aires, vol. 3, no 11, abril-junio 2005, pp. 37-51 <http://www.iigg.fsoc.uba.ar/elatina.htm>, [consulta 24 de octubre de 2005].
} 
Todas estas tendencias ofrecen la posibilidad de que la historiografía española se pueda pensar sin la habitual mediación de autores y escuelas de otros países y ser parte de la configuración de la recomposición de un nuevo paradigma y nuevas escuelas. Veamos, pues, el caso de una de ellas, el de Historia a Debate, la cual vamos a explicar un poco en detalle.

\section{LA PROPUESTA HISTORIOGRAFÍA DE HISTORIA A DEBATE}

La alternativa historiográfica de Historia a Debate descansa en tres pilares construidos a partir de las ideas de Tomas S. Kuhn ${ }^{49}$ y de la lectura que hace de ellas otro autor, Carlos Barros ${ }^{50}$. La síntesis del pensamiento de ambos autores podríamos denominarla como un nuevo pensador, que podríamos denominar Kuhn-Barros, con el fin de cerrar las puertas a un flanco de la crítica que incidiría en la cuestión de si la lectura de Carlos Barros sobre Kuhn es o no la correcta, o si utiliza tal o cual concepto de Kuhn en consonancia con sus teorías generales. Esta constatación hace que todos los posibles comentarios sobre los principios rectores de Historia a Debate tengan como referencia a esa nueva lectura o interpretación que ha realizado Carlos Barros sobre Kuhn, que porque no reconocerlo, puede ser un puente entre las teorías de éste último y Bourdieu. El estudio de Carlos Barros sobre Tomas S. Kuhn descansa en tres rectificaciones: a) Carlos Barros abre la "comunidad científica" a la sociedad, es decir, la entiende como algo sensible a los acontecimientos y no como un comunidad encerrada en sí misma y distante de su tiempo; b) introduce el consenso y el equilibrio en la forma en que avanza el conocimiento de la historia. La literatura kuhniana incide en el concepto de ruptura como principio rector del progreso científico, pero Carlos Barros, muy inteligentemente, introduce también el término de "continuidad", con el fin de concebir la evolución del conocimiento científico no sólo como una "ruptura" con lo anterior, sino también como una "continuidad" con el legado científico. Por tanto, Carlos Barros afirma que el conocimiento histórico avanza gracias a la "continuidad y ruptura"; c) utiliza el concepto de "paradigma" en su significación clásica, aunque flexibiliza su conte-

\footnotetext{
${ }^{49}$ Iggers fue uno de los que introdujo sus ideas en la historia. Ver IGGERS, G. G., New directions in European historiography, Wesleyan University Press, Conneticut, 1984. Para profundizar más en la relación del concepto de paradigma y su relación con la historia de la historiografía, ver: PASAMAR, G., "El concepto de paradigma y su importancia en historia de la historiografía", en Miguel A. Cabrera y Marie McMahon (coords.), La situación de la Historia. Ensayos de historiografía, Universidad de La Laguna, Tenerife, 2002, pp.135-155.

${ }^{50}$ Profesor de la Universidad de Santiago de Compostela y Coordinador de la Red Internacional de Historiadores Historia a Debate. Especialmente en sus trabajos y como coordinador del grupo de trabajo multinacional e intra y pluridisciplinar que elaboró el Manifiesto historiográfico Historia a Debate.
} 
nido. Así el "paradigma" de Carlos Barros también hace referencia a los consensos inadvertidos y a los preceptos mínimos que comparte la comunidad de historiadores.

Estas tres rectificaciones de Kuhn explican la arquitectura teórica de la alternativa historiográfica de Historia a Debate, pero no la comunidad internacional de historiadores, ni el éxito de ambas. La enorme difusión de Historia a Debate como comunidad internacional de historiadores y de su alternativa historiográfica se debe a la necesidad de un cambio en la disciplina de la historia y a la búsqueda de un nuevo paradigma que sustituya al anterior ${ }^{51}$. La búsqueda de este cambio queda reflejada en el resultado de la encuesta internacional "El estado de la historia" ${ }^{52}$, que fue enviada a más de 30.000 historiadores de todo el mundo ${ }^{53}$.

Una vez establecidos los pilares teóricos en los que se sustenta la alternativa historiográfica de Historia a Debate, y la constatación de que la comunidad de historiadores demanda un cambio, vamos a fijar los elementos fundamentales de la comunidad científica de Historia a Debate.

1) La Comunidad. La Comunidad. Historia a Debate es una comunidad red con interminables nodos en universidades de los cinco continentes. Es, en definitiva, una comunidad académica de nuevo tipo implicada con la historiografía, la metodología y la teoría de la historia y con la información crítica, al mismo tiempo que es solidaria y, lo que es más importante, tiene una identidad común, que se puede atribuir tanto a unos intereses culturales compartidos como a la identificación con un proyecto conjunto. Este carácter identitario lo conforman tanto la comunidad virtual ${ }^{54}$ como real de historiadores que están

\footnotetext{
${ }^{51}$ Proceso que se producirá en acuerdo con la dinámica "continuidad/ruptura" señala más arriba.

${ }^{52}$ La encuesta internacional "El estado de la historia" es el resultado de dos proyectos de investigación coordinados y dirigidos por el profesor Carlos Barros desde el año 1996 al 2001. Su equipo, objetivos y resultado se pueden consultar en www.h-debate.com/encuesta/ menu.htm

${ }^{53} \mathrm{La}$ encuesta consta de más de ochenta cuestiones donde se pregunta a los historiadores tanto por el estado de la disciplina como por su pasado y futuro. De ella se concluye que son necesarias variaciones en la configuración teórica, filosófica y metodológica de la disciplina con el objetivo de crear un nuevo paradigma, que ofrezca tanto lo mejor de lo "viejo" como de lo "nuevo". Asomándonos un poco a los resultados del cuestionario se puede observar que los historiadores que han contestado consideran que la disciplina de la historia sí está en crisis, y un 56\% por ciento consideran que la salida a ese estado sería la elaboración de un nuevo paradigma. En el mismo sentido, son mayoría los que opinan que los valores compartidos por los historiadores tienen mucha importancia, con lo que podemos establecer sin equivocarnos que entre los historiadores hay un gran sentido de comunidad. Por otro lado, un $84 \%$ cree que Internet influye mucho en la escritura de la historia. Como resultado podemos concluir que vuelven a estar presentes en la opinión de los historiadores términos como "nuevo paradigma" y sentido de comunidad. Para más información, ver: www.h-debate.com

${ }^{54}$ Entiéndase "virtual" en el sentido de que está configura a partir de una red digital y no en el sentido de sustitutivo de la realidad.
} 
en contacto en la red internacional de historiadores Historia a Debate, tanto diariamente a través de Internet como presencialmente en los Congresos, conferencias y presentaciones organizadas por miembros de la Comunidad. Como consecuencia del carácter doble de interacción científico de los historiadores -en Internet y presencial- se pueden establecer tres círculos de participación en la comunidad. El círculo más externo o "blando" es el que recibe las comunicaciones de las actividades de Historia a Debate y se comporta de una forma pasiva; el círculo próximo sería aquel que está suscrito a las listas y participa en los Congresos en una actitud activa-pasiva en función de sus intereses; y por último el núcleo "duro" o círculo más próximo que está más implicado en la elaboración de la alternativa historiográfica y forma parte de proyectos y actividades de Historia a Debate. El carácter de comunidad red ofrece la posibilidad de que los historiadores puedan saltar de un círculo a otro dependiendo de la actividad a desarrollar según sus intereses y disponibilidad.

2) El debate. El debate no es un uso académico, pero es una actividad que ofrece "continuidad" a los períodos de ciencia normal y que ofrece la posibilidad de la sociabilidad académica ${ }^{55}$. Los debates se producen diariamente a través de Internet en las listas de Historia a Debate y su página web, y a lo largo de los Congresos. Los debates tienen una estructura formal. Alguien plantea una cuestión, que es rebatida en un intercambio dialéctico donde siempre una postura ofrece menos contradicciones que su oponente y la supera, y ésta es a la vez derrotada por nuevas visiones que superan a las anteriores. Y así sucesivamente. Esta actividad es la verdadera expresión de las denominadas "cientific wars", que provocan conflictos dentro de la comunidad y que son las responsables del avance de la disciplina.

3) Red. La red Historia a debate está conformada por un conjunto de vínculos entre diversos nodos articulados y localizados en diferentes espacios académicos y comunitarios. La actividad empieza en un nodo central y se amplia indefinidamente de tal manera que ese punto central puede mudarse a cualquier nodo en un momento determinado por alguna necesidad. Este concepto de red tan disperso y descentralizado hace llevar a una concentración mínima que implica una gran solidez. En el mismo sentido, esta configuración supone la puesta en marcha de nuevas formas de trabajo, como el trabajo en red.

4) Carlos Barros. Carlos Barros es una de las piezas angulares de la Comunidad y Red de historiadores Historia a Debate en su papel de coordinador intelectual y

${ }^{55}$ Este es otro elemento clave en la configuración del pensamiento de ese nuevo teórico llamado Kuhn-Barros. 
científico, así como editor y moderador de los debates y de la página de Historia a Debate y de su propia página personal ${ }^{56}$. La importancia del profesor Carlos Barros en las actividades de Historia a Debate no se debe confundir con la necesidad de muchos historiadores de fabricar un nuevo "gran hombre" que lo hace todo. Su presencia y dinamismo son imprescindibles para Historia a Debate pero sin el trabajo, la dedicación y la ayuda de todos los nodos de la red y todos los miembros de la comunidad, simplemente no existiría Historia a Debate.

Estos cuatro elementos son algunos de los que configuran la comunidad de historiadores Historia a Debate, que se ha ido desarrollando desde su nacimiento en 1993, fecha desde la cual podemos establecer tres etapas en la trayectoria de Historia a Debate. Veamos su desarrollo.

a) Etapa de los Congresos (1993-1999). Esta fase está íntimamente ligada a la organización, desarrollo, conclusiones y publicación de los resultados de los dos congresos internacionales Historia a Debate. Se puede considerar como el período más convencional de la Comunidad, aunque los congresos de Historia a Debate no se pueden considerar nada ortodoxos, puesto que la introducción continua de debate los hace diferentes. El resultado de ambos Congresos ha quedado reflejado en las actas de los mismos (seis tomos del primer Congreso y tres del segundo). Si el I Congreso Internacional Historia a Debate celebrado en el año $1993^{57}$ fue una "toma de contacto" con la situación de la historia y con el estado de las grandes escuelas (Annales, Past and Present, etc.), el II Congreso Internacional Historia a Debate ${ }^{58}$ significó la posibilidad de comenzar a plantearse problemas y resolver cuestiones de una forma autónoma, así como la oportunidad de iniciar un proceso de pensar con cabeza propia tanto el pasado como el presente y futuro de la disciplina de la historia. Además, por primera vez, la comunidad de historiadores latina tenía la oportunidad de tener voz en el debate historiográfico internacional ${ }^{59}$. La relación de la historia con la literatura, la teorización sobre la cientificidad de la historia y la relación de la historia con la sociedad fueron tres

\footnotetext{
${ }^{56}$ La página de la comunidad internacional de historiadores Historia a Debate es la del profesor Carlos Barros es http://www.cbarros.com

${ }^{57}$ Se desarrolló durante los días 14-18 de julio de 1993 en el Auditorio de la Universidad de la Universidad de Santiago de Compostela.

${ }^{58}$ Tuvo lugar del 14 al 18 de julio de 1999 en el Palacio de Congresos y Exposiciones de San Lázaro en Santiago de Compostela.

${ }^{59}$ Argentinos, cubanos, españoles, mexicanos y otros miembros de otros países latinoamericanos pudieron exponer sus inquietudes y propuestas a la par de franceses, ingleses y norteamericanos. Ver programa del II Congreso Internacional Historia a Debate en la página web y en las Actas del mismo.
} 
de los grandes temas de los 236 resúmenes presentados en el Congreso ${ }^{60}$. En la organización y desarrollo del II Congreso se introduce por primera vez Internet en Historia a Debate.

Además de los Congresos, se puso en marcha el seminario internacional Historia a Debate, que desde 1997 ha reunido en Santiago de Compostela a 27 invitados de universidades europeas (Francia, Austria y Portugal), latinoamericanas (Argentina, Brasil, México, Cuba y Perú), norteamericanas (Canadá) y de Oceanía (Nueva Zelanda). Además de estas sesiones realizadas con invitados "externos", el seminario se reunió mensualmente desde su inicio hasta el año 2002 alternando las sesiones con invitados con las "internas" realizadas por los miembros permanentes del seminario. El seminario pasa a ser "on line" desde el 2002, año a partir del cual las sesiones presenciales se van intercalando más en el tiempo debido al contacto diario por medio de Internet ${ }^{61}$. Las temáticas de los seminarios han sido, y son, muy variadas, combinando sesiones teóricas e historiográficas con otras centradas en cuestiones más empíricas, aunque siempre intentado mostrar su perfil metodológico.

b) Etapa de Internet, que abarca desde el año 1999 al 2001. Esta fase comenzó, como se ha indicado, con la organización del II Congreso Internacional Historia a Debate. Durante este tiempo y hasta el año 2001 Historia a Debate se transforma en una comunidad académica de nuevo tipo, que quiere decir que se van conformando los cuatro elementos que se han citado más arriba (comunidad, debate, red y coordinación) y se convierte en una red estable de funcionamiento diario desde los cinco continentes. Unos meses después de la conclusión del II Congreso, nace la lista Historia a Debate, que tiene como miembros a los inscritos al citado Congreso más una serie de profesores que se les informaba de los seminarios y de otras actividades y noticias de Historia a Debate. El primer mensaje de la lista es del día 23 de septiembre de 1999. Las comunicaciones crecieron de manera exponencial a lo largo de los años. En 1999 hubo 177 mensajes; en el 2000, 1052; en el 2001, 1651; en el 2002, 1176; y en el 2003, 1500. En todos estos años hubo un momento delicado cuando en noviembre de 2001 el coordinador

\footnotetext{
${ }^{60}$ Ver libro de Abstracts del II Congreso Internacional Historia a Debate. Abstracts del II Congreso Internacional Historia a Debate, Santiago de Compostela, 1999.

${ }^{61}$ Las distintas sedes del Instituto de Estudios Gallegos "Padre Sarmiento" (CSIC) acogieron al seminario desde su nacimiento, en 1997, hasta el año 2003, fecha en la que se traslada a la Universidad de Santiago de Compostela.
} 
toma la decisión de reestructurar el curso de los debates, por un cierto agotamiento de los mismos, y decide suprimir los mensajes anónimos y aquellos que impliquen falta de respecto a uno o varios interlocutores. Como consecuencia de este hecho, a partir de ese momento todos los mensajes tendrían que ir firmados y ser respetuosos con los otros participantes en los debates. La regeneración de los debates en curso y la apertura de nuevos temas fueron el resultado de estas decisiones, que supusieron una reactivación de la comunidad. Pese a ello hay que reseñar que seguía habiendo demasiados mensajes "amén"62, y también hay que destacar, pero de una forma positiva, el poco protagonismo del moderador y coordinador de Historia a Debate, Carlos Barros.

La lista Historia a Debate se vio complementada desde el 1 de enero de 2001 con la lista de Historia inmediata, gracias a un mensaje del profesor Juan Paz y Mino, de la Universidad Pontificia de Ecuador, sobre una revuelta indígena en su país. Este mensaje fue simplemente un pretexto para poner en marcha una lista que venía funcionando en paralelo con la de Historia a Debate casi desde su inicio, y a través de la cual se hacían girar llamados y manifiestos de compromiso y denuncia. La conformación de esta lista ofreció la posibilidad de observar de una forma privilegiada el cambio que estaban suponiendo las nuevas tecnologías para la escritura y la percepción de la historia. Se estaba pasando de la figura del historiador como un juez alejado de su objeto de investigación, a un historiador nuevo que a la vez que vivía la historia la escribía, es decir, era sujeto de lo que estaba viviendo y lo estaba escribiendo, con lo que podemos afirmar que estábamos pasando a una nueva dimensión de la profesión del historiador, ahora convertido en "ciudadano historiador" o "historiador participativo". Además del caso de Ecuador, se pudo comprobar lo mismo con los acontecimientos de Venezuela o Argentina.

Las características de estas dos listas son muy evidentes. El porcentaje de participantes activos en las listas eran menor del 10\%, algo no muy diferente de lo que es común en las diferentes listas académicas del mundo. La otra cara de ese porcentaje de miembros activos son los pasivos, que crean auditorio, aunque sea un auditorio silencioso, estimula la participación activa porque concede prestigio, favorece la interacción social, la información y la conformación de una comunidad virtual, que lleva a compartir preocupaciones y problemas, a hacer uso común del mismo medio y a establecer un trato especial entre miembros.

\footnotetext{
${ }^{62}$ Aquellos que se limitan a dar la razón a una línea de argumentación sin aportar nada.
} 
Además de las dos listas está la página web, que se cuelga en la red el 29 de abril de 1999 y que ha tenido un desarrollo espectacular tanto a nivel de contenido como en visitas ${ }^{63}$. Los países que más visitan la web son EE.UU, México, Argentina, España, Perú y Chile. Además en la web, se pueden encontrar los diferentes debates de las litas que se centran en historiografía, enseñanza, teoría e historia inmediata. Por último, la web tienen un consejo consultivo compuesto por un total de 30 miembros, donde dominan los historiadores españoles, mexicanos y estadounidenses.

c) Etapa de conversión en tendencia historiográfica. Esta fase comienza con la elaboración y posterior publicación del Manifiesto historiográfico Historia a Debate, que ha sido traducido a ocho idiomas, que en la actualidad cuenta con más de 400 adhesiones de más de 50 países, y que apuesta por una historia con sujeto, por el entusiasmo del historiador, por la innovación metodológica e historiográfica y por el compromiso con el tiempo en que vivimos. El manifiesto ha sido publicado más de 40 veces en sus diferentes idiomas tanto digitalmente como en papel ${ }^{64}$. Asimismo, Historia a Debate como tendencia historiográfica ha dado lugar a la cristalización de un núcleo duro de historiadores que están en contacto mediante una tercera lista, que se denomina "Grupo Manifiesto" y que gestiona personalmente en el coordinador de Historia a Debate, Carlos Barros.

La tendencia historiográfica de Historia a Debate se ha visto notablemente consolidada con la muy favorable acogida que ha tenido la llamada "convocatoria específica" 65 en el III Congreso Internacional Historia a Debate, que iba a dirigida tanto a estudios e investigaciones partiendo de los presupuestos de Historia a Debate como a trabajos centrados en la propio historia de Historia a Debate. Este proceso se verá impulsado nuevamente con el desarrollo del los grupos de investigación en red que se están preparando ${ }^{66}$.

\footnotetext{
${ }^{63}$ En mayo de 2000 eran de 75 visitas al día y 2354 al mes. En enero de 2001 eran de 132 al día y 4099 al mes; en enero de 2002 eran 428 al día y 13275 al mes; en enero de 2003 874/271222, y en abril de 2155/64653, con un total de más de un millón y medio de visitas en la actualidad. Ver http://www.h-debate.com/Spanis/estadisticas/menu1.htm

${ }^{64}$ En Santiago de Compostela ya se han realizado cuatro ediciones. Además ha sido presentado en más de 50 actos en diferentes universidades de Europa y América. Ver sección de presentaciones en www.h-debate.com/Spanish/presentaciones/menu.htm

${ }^{65}$ Ver en la página web de Historia a Debate en el apartado correspondiente al III Congreso Historia a Debate, convocatoria específica. Ver http://www.h-debate.com/congresos/3/menu.htm

${ }^{66}$ Y que se están desarrollando en el marco de la Acción especial "Red Temática Historia a Debate”, desarrollada con apoyo del Ministerio de Educación y Ciencia.
} 
También hay que añadir a esto el proyecto de Academia Solidaria, que ha servido para que los historiadores de todo el mundo tomen postura sobre cuestiones actuales, como ha sido el caso del profesor argentino Raúl Dargoltz ${ }^{67}$. Con esto queda claro el nuevo tipo de compromiso intelectual que se intenta fomentar desde Historia a Debate, un compromiso más con el tiempo y con las personas y con la historia que con un partido político o unas ideas.

Para cerrar este apartado, se pueden enumerar una serie de características propias del proyecto historiográfico Historia a Debate, a saber: a) foro y tendencia historiográfica son inseparables; b) mutifocalidad y simultaneidad; c) es un proyecto de carácter latino; d) proporciona un intercambio igual y una multilateralidad en las reflexionaes; e) fomenta el trabajo en red; f) pone de relieve la importancia de Internet; g) colabora en la formación de la globalización alternativa gracias a sus posturas críticas; h) es un proyecto colectivo y participativo; i) aporta los testimonios directos, lo que hace que el historiador sea a la vez testigo, cronista y analista (al contrario del periodismo digital, que sólo cuenta de forma inmediata; j) compromiso con el tiempo y con la profesión como parte esencial del proyecto; k) es un movimiento historiográfico; 1) trabaja con varias ideas a la vez; m) se abandona la idea de idealizar al autor y a la obra; n) es una comunidad en red virtual y real; ñ) es plural, complejo y global.

\section{EL FUTURO DE LA HISTORIOGRAFÍA ESPAÑOLA}

Con lo expuesto hasta aquí se puede concluir que la historiografía española ha desarrollado un notable auge, un gran dinamismo y una considerable autonomía, teniendo en cuenta la situación de atraso de la que partía ${ }^{68}$. Este auge tiene una serie de planos que pasamos a desgranar a continuación:

\footnotetext{
${ }^{67}$ Ver todo el proceso de Raúl Dargoltz en: Para la Academia Solidaria, consultar http://www.hdebate.com/Spanish/academiasolidaria/menu.htm

${ }^{68}$ En este sentido es necesario considerar la apreciación que hace Gonzalo Pasamar al respecto: "En nuestro país la renovación historiográfica, salvo ciertos sectores en los que se ha producido una expansión novedosa de la profesión en los años sesenta, es un proceso que ha carecido apenas de rupturas y donde las influencias europeas han ido recibiéndose de un modo muy personalizado y disperso. Esta constatación no entraña, en principio, un juicio de valor ni positivo ni negativo". PASAMAR, G., "Maestros y discípulos: algunas claves de la renovación de la historiografía española en los últimos cincuenta años" en La historia local en la España contemporánea. Estudios y reflexiones desde Aragón, Universidad de Zaragoza, Zaragoza, 1999.
} 
a) La historia, además de ser hija de la sociedad que la crea y la cultiva ${ }^{69}$, no puede formular sus cuestiones básicas sin la ayuda conceptual y narrativa de la dimensión historiográfica, teórica y metodológica ${ }^{70}$.

b) La plena integración internacional de la historiografía española, basada en el intercambio, requiere en resumidas cuentas una mayor atención a la investigación de la historiografía española más reciente ${ }^{71}$.

c) La importancia de la teoría y de la integración internacional son matizadas por el contexto actual, en el que la investigación histórica, al descentralizarse y ampliar su campo de acción, ha conseguido reducir su dependencia respecto a las instituciones, con lo que las escuelas nacionales han dejado de tener tanto peso como el que tenían ante el empuje de la globalización y la desinstitucionalización ${ }^{72}$ (esto ha hecho posible la aparición de alternativas como la de Historia a Debate.

d) Internet y las nuevas tecnologías actúan como contrapeso horizontal y transversal de la verticalidad y la compartimentación de las viejas formas de asociación y comunicación académicas, que se caracterizan por su jerarquía y lentitud. Por otro lado, la comunicación a través de Internet también facilita el trabajo en red, que representa la posibilidad de poder trabajar e investigar de forma colectiva ${ }^{73}$.

e) Internet, la globalización académica y las nuevas tecnologías ha hecho posible la aparición, desde España, de nuevas realidades y tendencias historiográficas, como Historia a Debate, que partiendo de un pasado historiográfico más receptor que emisor de novedades ha logrado construir y proyectar una alternativa historiográfica de ámbito académico latino. Esa característica, sumada a la historia y lengua comunes, son suficientes motivos para una

\footnotetext{
${ }^{69}$ Ver PEREIRA-MENAUT, Gerardo, "Formación técnica vs. Humanismo. Aproximación crítica", Mientras Tanto, n 68/69, 1997, pp. 146-148.

70 "...querer hacer historia sin hablar de ella es una propuesta suicida". Ver BERMEJO, J.C., "Hacer historia, hablar sobre historia", Actas II Congreso Internacional Historia a Debate, Santiago, 2000, p. 21.

${ }^{71}$ Ver BARROS, C., "Inacabada transición de la historiografía española", Bulletin d'Histoire Contemporaine de l'Espagne, $\mathrm{n}^{\circ}$ 24. Bourdeaux, décembre 1996, pp. 469-493.

${ }^{72} \mathrm{La}$ historia se ha revelado como una realidad compleja y han proliferado los debates teóricos que han resultado vitales para la revitalización de la disciplina y el recurso a las terceras vías. Ver Aurell, Jaume, La escritura de la memoria. De los positivismos a los postmodernismos, Universidad de Valencia, Valencia, 2005, pp. 199-210.

${ }^{73}$ Aunque sigue siendo imprescindible la actividad presencial, ver BARROS, C., "Historia a Debate, tendencia historiográfica latina y global", Aula-Historia Social, Valencia, no 13, primavera 2004, pp. 84-90.
} 
fuerte interrelación universitaria que ha dado origen a esta alternativa. Historia a Debate, es síntoma, causa y consecuencia, de la descentralización historiográfica provocada por una globalización diversa que está dando a luz una nueva historiografía. Trastocados los viejos centros y periferias historiográficos, Historia a Debate propone y practica un nuevo modelo de relaciones historiográficas internacionales en consonancia con el tiempo presente, cimentado en el intercambio igual, el multiculturalismo historiográfico y el trabajo en $\mathrm{red}^{74}$.

f) Historia a Debate, y por tanto la historiografía española, tienen una creciente aceptación en el ámbito académico internacional ${ }^{75}$.

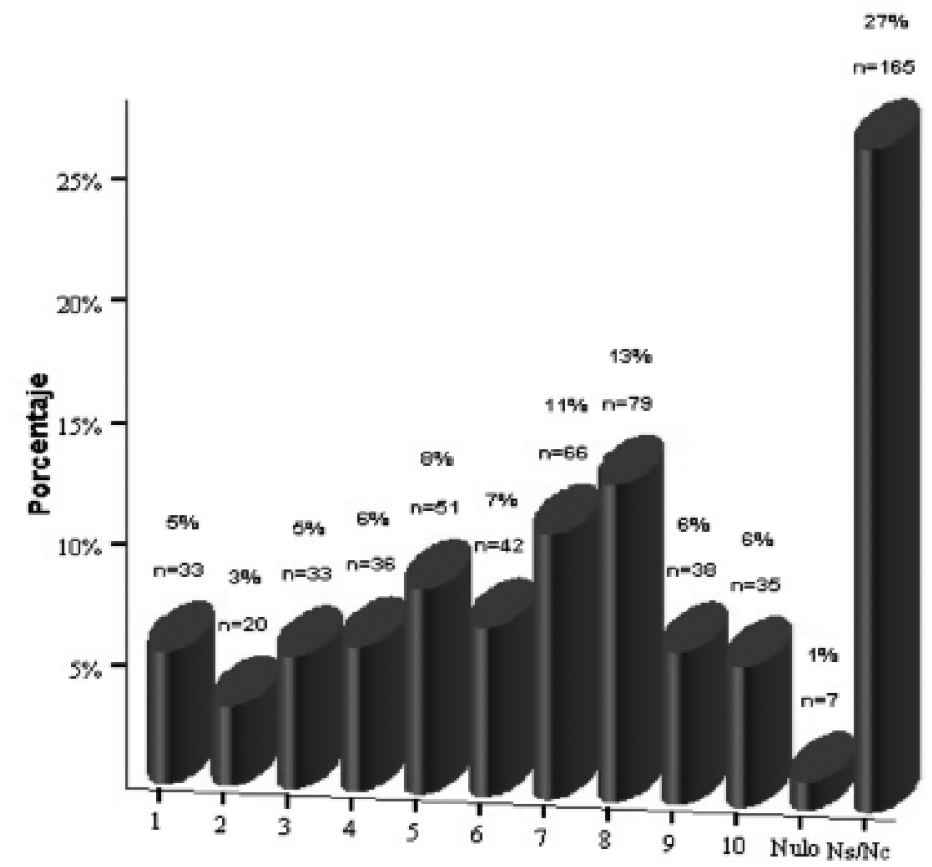

Nủmero total de casos: 605

${ }^{74} \mathrm{El}$ ámbito latino fue lugar de recepción de las nuevas historias annalistes y marxistas y que ha facilitado la posibilidad de unas síntesis que no se dieron en sus focos de origen.

${ }^{75}$ Como de muestra el libro History under Debate. International Reflection on the Discipline, The Haworth Press, New York, 2004. 